\title{
Research on Tourism of Major Sports Event
}

\author{
Tao Zhang \\ Nanyang Institute of Technology, Nanyang, Henan, 473004
}

\author{
Keywords: Tourism Research, Sports Event, Efficient Development
}

\begin{abstract}
There is no complete theoretical framework for the study of the interaction between major sporting events and urban tourism. Since both are the industries that the country is committed to developing, the relevant theoretical research is more urgent as the advantages of interaction are becoming more and more prominent. This paper starts from the essence of the major sporting events and the urban tourism, tries to explore the general pattern of the interaction process and the interactive mechanism that supports the interactive interaction and the win-win cooperation by means of the literature research method, the historical research method and the interdisciplinary approach. On the basis of constructing the framework of benign interaction mechanism, this paper analyzes the problems existing in the process of interaction between major sports events and urban tourism, and discusses the relevant countermeasures which are suitable for the interactive development of the two.
\end{abstract}

\section{Introduction}

The city is facing competition, so the city tap their own potential to enhance its political, economic, cultural strength is imminent. Each city can be seen as a national brand, with the process of globalization, how to expand the impact of the city, what kind of carrier to achieve the city marketing, it can be said that as an important carrier of sports major sports events is one of the one. The combination of major sporting events and modern media technology allows the world to remember these cities and let the world appreciate the unique image of these cities. The city as an important part of human civilization, each city has distinctive characteristics, and urban tourism is also a city of human landscape and natural landscape to attract elements and developed a unique way of tourism. For example: Beijing's courtyard, modern civilization and political culture; Xi'an after 13 ancient capital, has a strong imperial charm; Shanghai is China's largest commercial, financial center and so on. On the other hand, the organization of major sporting events has a positive effect on urban tourism, affecting the politics, economy and culture of a city. In addition, the sports activities of the city have been closely related to the urbanization of the city. The event will have an impact on the urban ecological environment, through the sporting events on the city to transform the city's ecological environment problems are inevitable; Finally, tourism is an experience, sports events brought about by this experience and personal And even the country's economic level is proportional to people in this experience will bring spiritual and physical health and beauty, this experience behind the hidden behind the growing socio-economic background, so in the final analysis, it is because of economic growth , People's living standards improve, have this experience, and in turn promote the new growth of the national economy.

China's major sports events and urban tourism interaction research is still in its infancy, the lack of major sports events and urban tourism interactive development research, this article for major sports events and urban tourism interaction in the process of interaction, impact, constraints and interactive mode, interactive Mechanism and other practical issues to study, to further explore the major sports events and urban tourism interaction has a certain theoretical significance.

\section{Analysis on the Characteristics of Major Sports Events and Urban Tourism}

Major events have a high degree of aggregation, Baines and Muir Si studied the special events of the "peak phenomenon", that special events can produce their own related services and generate 
economic benefits. As a large number of audience participation and multi-channel media communication, once the major sports events, related to the specific environment can gather significant flow of people, logistics, information flow, capital flow, the formation of collective effect. Fan Zhen in the "ignite the Olympic industry" that the sports industry's aggregate effect is mainly reflected in three aspects: First, a large number of people gathered space, bringing a lot of related services industry demand, resulting in comprehensive economic benefits; Second, a lot of information The flow of time gathering, gathering and event-related concerns, to promote the exchange of ideas and values of different ideas and values, the resulting intangible value is very impressive; Third, a large number of logistics space gathered, including athletes, spectators, media and business-related Equipment and materials. In some cases, the collection effect of major sporting events is also two-sided, manifested as the aggregation of economic, will cause many negative impact on the host.

On the economic properties of sports events, many scholars believe that sports events are "experience the economy." From the consumer point of view, the consumption of sporting events is entertainment and leisure consumption, the audience to buy events and souvenirs not only pay for pleasure, but also for the experience to pay, so the sporting event consumption has a significant service economy experience economy can be defined For the cultural experience industry. Therefore, the major sporting events can have a strong emotional ease, infection and interactive effect. This remarkable experience of the characteristics, making a lot of fans to watch the event bored, watch a high level of events, especially a kind of enjoyment. This is also the city held a major sporting events, attracting tourists to increase urban passenger flow, improve tourism income, and ultimately promote the development of tourism economy is the key.

Sports events originated in the early human ritual activities, with the development of social productive forces, with the progress of human civilization and progress, its own is a cultural phenomenon. The spirit of "mutual understanding, friendship, solidarity and fair competition" has become the common language of different ethnic groups in different ethnic groups. The spirit of "higher, faster and stronger" is the totem culture that is turned into human spirit. Human pursuit of excellence, challenge the limit, beyond the self.

Sports events are not the beginning of the industrial nature, this feature is with the special economic and social environment arising. Su Dongshui that the industry as a product of social division of labor, industry is also similar to the collection of economic activities, with the emergence of social division of labor generated, development and development of social productive forces is the result of continuous development. With the development of modern sports events, the provision of sporting events business together and have a huge impact on the formation of sports industry. There is no form of any kind of industry and sports events, from research and development to promotion, from production to consumption, in series from the commercial chain of all links. With the growth of the economy, human development needs a higher level of spiritual pursuit, sports events can be derived from many industries, such as: sports economy, sports finance and insurance, sports media and advertising industry and so on.

The charm of major sporting events lies in its innovation, which is also its essential attribute. Different types of sports events are different, the same kind of sports events because of the natural environment, geographical conditions, human history and historical environment, the economic level and different. So the major sporting events always need to continue to innovate, so as to make the sports followers never tired.

\section{Analysis on the Interactive Mode and Mechanism of Major Sports Events and Urban Tourism}

The interaction between sporting events and urban tourism is complicated. Which involves a lot of the main body: influential sports events of the professional bodies, the competent departments, tourism enterprises and athletes and tourists and so on. The benign interaction between major sporting events and urban tourism needs to establish and perfect the sporting mode of sporting events in line with international practice. The two modes of interaction are: sports events and urban tourism. Under the management of professional management institutions, Marketing, integration of 
resources, complementary development, to achieve win-win situation. The steps of the interactive operation are as follows: (1) Under the overall plan of the higher authorities, the relevant sports institutions of the sports competitions shall formulate the annual event plan and submit them to the higher authorities in accordance with the arrangement time of the events and the activities of the events, Provide the necessary facilities and so on, and organize the associated enterprises to organize the event to provide a sound supporting services. (2) the marketing stage of sporting events, the relevant management departments and tourism enterprises to invest a lot of money and resources on the event, and actively implement the marketing strategy, make the greatest efforts for sports events and tourism to attract more tourists. (3) During the sporting event, the responsible tourism enterprises should provide a series of supporting services such as food, housing, shopping and entertainment for the participating and spectators. In this way, the enterprise can not only obtain more objective income, Is rich in a large number of tourists and athletes in the game of life. Good supporting services can enhance the overall image of the city's tourism industry, and even can be sustainable development, and this good momentum of development in turn conducive to sports events to attract more tourists, this is the process of interaction between the two win-win process The (4) after the end of the sporting event, the tourism sector and tourism enterprises to participate in and continue to tap the sporting event venues facilities tourism function, to achieve the event stadium maintenance and tourism node innovation win-win situation.

Major sports events and urban tourism interaction process need to learn from foreign advanced experience, the formation of interactive effects of scientific assessment, assessment system, assessment assessment elements should include economic, political, cultural, environmental and other aspects, through the assessment system to generate quantitative data on the one hand To provide a basis for the planning of major sporting events, on the other hand to form a scientific incentive system for different departments to encourage incentives for potential market participation projects, enterprises through incubation support and other means to form, including assessment system, reward system, System as the main content of the incentive mechanism to create a major sporting events and urban tourism benign interaction mechanism pattern.

Sports events are essentially through the operation of the final competition products and services output, in the process will inevitably exist in the consumption and product supply, so the major sporting events and urban tourism interaction process need to establish a sound security mechanism, including the legal system, policy And resource investment system, personnel training system and risk control system. Major sports events and urban tourism interaction process, not only with the tourism sector involved in contract negotiations, marketing market, sponsorship advertising, hospitality services and other aspects of the specific operational tasks, but also with other departments involved in fiscal revenue, television rights, insurance, audience litigation And so on, so the targeted legal system will be escort for benign interaction, and in increasing policy support, rational allocation of public resources and capital investment at the same time, should improve cross-sports, tourism and other departments of the relevant special talents to introduce, System, in addition, the potential risks of major sports events varied, must do all kinds of risk assessment, prevention and control system, fully do a good job of risk prevention at the same time, establish and improve all kinds of contingency plans.

Major sports events and urban tourism As the inherent attributes of the service industry, the behavior and demand of sports participants, event tourists and urban tourists constitute the core elements of the important sports events and urban tourism interactive innovation mechanism. For urban tourism, with the improvement of people's living standard, the improvement of spiritual and cultural needs, the continuous promotion of urban tourism products, the rational expansion of urban tourism space, urban tourism forms also emerged at different levels of change, from "day to see the temple, perfect sleep "A single form of evolution of science and technology tourism, exhibition tourism, health tourism and so on. At present, people demand for major sporting events and participation are also drastic changes, all of these changes in demand for major sports events and urban tourism derivative products toward the humane, personalized, multi-polar development, so only through the establishment of Innovation mechanism to meet the needs of the market, 
innovation mechanism, including both managers of ideas and innovative ideas, should also guide and encourage major sports events marketing model innovation and through the urban tourism resources, sports culture in-depth mining, continuous design, innovation More quality sporting events tourism products.

\section{Conclusion}

The main strategies to promote the benign interaction between major sporting events and urban tourism are: the top design level macro grasp the major sports events and urban tourism science interaction, healthy and sustainable development; establish and improve the major sports events and urban tourism interactive development system mechanism; rich major sports events And urban tourism interactive development of marketing tools to encourage market operation and legal framework within the marketing breakthrough and innovation; to promote the development of sports and tourism gathering, to build a number of sports events tourism gathering area, focus on creating a unique sporting event tourism , Sports and leisure tourism brand; scientific co-ordination of major sports events and urban tourism interactive development of the whole process.

\section{References}

[1] Huang Haiyan. The effect of sporting events on urban tourism: a structural equation model of residents' perspective [J]. Journal of Wuhan Institute of Physical Education, 2015 (01)

[2] Huang Haiyan, Xu Lin, Luo Lei, Ma Jie.Study on the Interactive Development of Sports Events and Shanghai Tourism [J]. Journal of Shanghai Institute of Physical Education, 2013 (01)

[3] CHAI Wang-jun, ZHAO Min.Study on the selection of large-scale sports events in Chinese cities based on urban marketing [J] .City Observation, 2012 (06)

[4] Tian Jing, Xu Chengli. Impact of large-scale sports events on urban development mechanism [J]. Journal of Beijing Sport University, 2012 (12)

[5] Pang Xuwei, Gao Wenqian, Zheng Yueping, Lou Shiyi, Liu Pei.Study on the Development Mode of Shanghai Large-scale Sports Events and Urban Tourism [J]. Journal of Sports Science and Technology, 2011 (06)

[6] Xu Yansong. The impact of large-scale sports events on the development of urban tourism and the negative impact [J]. Modern Economic Information, 2011 (20) 